\title{
EL PARAISO Y LOS CENTROS CEREMONIALES TEMPRANOS DE LA COSTA CENTRAL
}

\author{
Por:
}

Jorge E. T. Silva S.

Universidad Nacional Mayor de San Marcos

La presente nota se inspiró en los propios trabajos publicados por el arquitecto Williams, cuyos datos y planteamientos han clarificado mi concepción sobre el nacimiento de la arquitectura como expresión social, despertando a la vez dudas, como las expuestas aquí, que la investigación deberá resolver. 



\section{I. - INTRODUCCIÓN}

Uno de los aspectos más fascinantes es sin duda el estudio de los patrones y sistemas de asentamiento de los pueblos prehispánicos que ocuparon los Andes durante los dos milenios antes de nuestra era. Los esfuerzos hasta ahora realizados son meritorios toda vez que se han obtenido datos concernientes con la naturaleza de los poblados, la manera como se utilizó el espacio y porque se dispone de suficientes evidencias que revelan modelos que tipifican períodos y regiones (Willey 1953; Rowe 1967; Schaedel 1951; Hardoy 1964; Williams 1971, 1972, 1980, 1981, etc.).

Los modelos o tipo fósiles (Childe 1972: 24) responden conductas socialmente establecidas. Así, los componentes materiales aparecen como resultado de una acción deliberada de los integrantes de una comunidad. Numerosos investigadores han reconocido correspondencias directas de las instituciones sociales y la estructura de los asentamientos. Trigger (1968) las señala claramente al referirse a la construcción de los santuarios y templos de la civilización Khmer. Flannery (1976: 16), Winter (1976:25), Lathrap et.al. (1977) encuentran que la variación de las casas de una aldea reflejaría división laboral, status, ordenamiento social, etc. El arquitecto Carlos Williams es muy elocuente a este respecto al manifestar que "la estructura social puede ser leída en la textura urbana, en la trama de los usos del suelo y en la forma de los poblados... quiero decir que he pensado la arquitectura como expresión cultural, donde la variación de formas es significativa y donde la difusión de patrones arquitectónicos en el espacio significará difusión de ideas en el mismo ámbito" (Williams 1980: 370).

La arquitectura no es, pues, únicamente una secuencia fisonómica sin contenido, un proceso "en virtud de la forma y la relación de sus superficies y volúmenes..." (Fritz 1978: 40), sino también una concepción que se torna tangible en respuesta a reglas del intelecto humano.

En los últimos años nuestro conocimiento sobre el surgimiento de la arquitectura en los Andes se ha enriquecido por los estudios del arquitecto Carlos Williams, quien logró producir una sinopsis comprensible de los diseños arquitectónicos peculiares de la costa peruana, subrayando la espectacularidad del canon en forma de "U" o herradura. De acuerdo al Arq. Williams (1980: 390, 421; 1981: 103, 109) el sitio arqueológico de Chuquitanta o Paraíso (valle del Chillón) constituye una de las manifestaciones más tempranas del patrón arquitectónico previamente señalado.

En las páginas siguientes revisaremos la asignación del Paraíso al Patrón en "U" por cuanto en nuestra opinión su morfología no se ajustaría a dicha configuración. Por cierto esta apreciación no es nueva (véase Scheele 1970; Moseley 1975). Sin embargo, hasta la fecha no hemos asistido a los deslindes de ambas posiciones. Por consiguiente el presente artículo, recogiendo los postulados de Scheele, Moseley (obras citadas) y sin intentar acaudillar una tercera alternativa, tiene la finalidad de reabrir y propiciar el debate en torno a este tema, no por un mero ejercicio intelectual del autor, sino porque, en primer lugar, Paraíso, un sitio sumamente complejo desde el punto de vista de su crecimiento, ha sido poco estudiado pero constantemente, y como antilogía, aparece en cambio citado en la bibliografía especializada; en segundo lugar, porque si se demuestra que la construcción de este sitio estuvo ligado a una concepción distinta al diseño en "U" entonces sería producto de la decisión de un comportamiento social diferente, lo que significaría ubicarse al margen de la superestructura (léase aparato ideológico, político y religioso) asociado al modelo en "U".

\section{II.- El SITIO}

El Paraíso o Chuquitanta se ubica en la margen sur del río Chillón, a unos $5 \mathrm{~km}$ al Este del mar. F. Engel $(1966,1967)$ visitó el lugar en los 50 excavándolo en 1965. Otros investigadores también se han ocupado de este sitio pero sin llegar a excavarlo extensivamente (Lanning 1967; Scheele 1970; Willey 1971; Moseley 1975; Williams 1971, 1980, 1981; etc.).

Engel se concentró en la Unidad I (Fig. 2) pero además hizo sondeos en las otras uni- 
dades. Según Engel (1966) se compone de 8 grupos de construcciones, número que podría ser mayor en opinión de Moseley (1975). La mampostería del lugar presenta muros de piedras irregulares y aristas cortantes, unidas con barro, recubiertas con un enlucido de barro y luego pintados de rojo, ocre, blanco y negro (Engel 1966, 1967).

\section{III.- El Paraíso: Planteamientos sobre su MORFOLOGÍA Y FUNCIÓN}

E. Lanning (1967: 70, 71) sostenía que los tres edificios principales del Paraíso se disponían de modo similar a los centros ceremoniales de la Costa. Evidentemente se refería a la concepción en "U" cuando decía "un templo central flanqueado por dos alas" que encerraban un gran patio abierto en el lado opuesto al templo. Más tarde, G. R. Willey (1971: 97) compartía dicha impresión puesto que sus notas se basaban en Lanning y Engel, aunque este último tenía una visión diferente.

En 1970, H. G. Scheele (1970) refutaba a Lanning argumentando que en el Paraíso no aparece patrón en $\mathrm{U}$ alguno por no existir una relación continua de los edificios y se suponía que era Precerámico.

Bajo esta perspectiva, en 1971, C. Williams (1971) incluyó a Chuquitanta en el modelo arquitectónico en forma de "U" ubicando "entre Mala al sur y Supe al norte" (Williams 1981: 95; Williams: 1980: 390) y tomándolo como ejemplo elaboró una secuencia del diseño en U situando a este sitio en la fase inicial de dicho patrón (Williams 1981: 101, 103, 109).

Sin embargo, persiste la posición contraria sustentada esta vez por E. M. Moseley (1975: 99) quien anota que la forma en $U$ de este sitio es fortuita y no deliberada, explicando en otros términos su naturaleza formal y funcional.

\section{IV.- Discusión}

En la introducción de este corto ensayo habíamos expresado nuestro deseo de propiciar un debate sobre la morfología de Paraíso. Por eso, retomamos la posta dejada por M. E. Moseley (1975) sin que ello signifique que coincida necesariamente con sus ideas sobre el surgimiento de la civilización en general o sobre el rol de Paraíso en particular.

De acuerdo a Williams el diseño en U se distingue porque "La pirámide principal está en el fondo de la $\mathrm{U}$ y compuesta a su vez por tres cuerpos: el central más elevado y dos laterales más bajos" (el subrayado es mío). Afirma igualmente (Williams 1980: 401, 402, 403) que "La plataforma" o "montículo" es seguramente la forma arquitectónica básica de la edificación andina", ilustrando en la figura 2.1. "algunas de las sendas de esta evolución formal" a base de 9 tipos que evolucionan desde al (montículo simple) hasta d4 (montículo con corredor central). En esta secuencia "El tipo (c2) es un montículo con alas laterales -léase a los costados- que evoluciona hacia (c3) y aparece como cuerpo central de los grandes conjuntos de planta en U o en herradura". Al agregar más elementos de juicio para identificar el patrón en U asevera "La pirámide principal está formada por un núcleo y una o dos alas laterales de menor altura que el núcleo. El núcleo es seguramente el edificio más importante del complejo y dentro del núcleo el atrio es el elemento más significativo" (los subrayados son míos) (Williams 1981: 97). Reconoce la importancia del núcleo cuando afirma "era un área de acceso restringido. El único camino para acceder hasta allí atraviesa el vestíbulo, la escalera, el atrio y nuevas escaleras. Es un buen lugar, con horizonte amplio, para la observación astronómica" (Williams 1981: 100). Este constituye consecuentemente el punto principal en donde se produjeron las actividades rituales y de culto. En Chavín de Huántar, Ancash, se aprecia esta jerarquía en sus dos períodos. El más antiguo "esconde" al Lanzón. El más 4reciente sirve de fondo al atrio de las lápidas y en su base oriental se halla la portada albinegra o de las falcónidas (Lumbreras 1970). El templo medio del cuerpo central de Garagay, Lima, está bellamente decorado con representaciones modeladas a mano y pintados (Ravines 1975; Ravines e Isbell 1976). Aquí, el cuerpo central sobrepasa en altura a los laterales.

De manera, pues, que el cuerpo central o fondo de la U debe ser anterior a las alas, constituyendo la edificación inicial que se amplió 
luego. Quizá por eso las estructuras laterales son a veces más bajas puesto que el central, por ser de mayor antigüedad y el área ceremonial principal, recibió sucesivas modificaciones, sea para reparar o rejuvenecer los recintos o para llenarlos y construir otros nuevos.

Por lo anotado, nuestra discrepancia tiene que ver con lo morfológico. Chuquitanta, como se dijo ya, se compone de 8 ó 9 grupos de construcciones que cubren unas $60 \mathrm{Ha}$. (Engel 1966; Moseley 1975). Algunos se emplazan en la base de las colinas adaptándose al relieve y la topografía, pero los montículos II y VI de Engel (Fig. 2) surgen de la parte llana y el VII se halla en la margen norte del Chillón. II y VI son considerados por C. Williams como las alas que configuran el modelo arquitectónico en U.

Observando los croquis de Engel, las fotos aéreas y al sitio en el propio terreno no existiría una deliberada disposición en U por las siguientes razones:

a.- Los montículos II y VI de Engel (1966) y que Williams ilustra (1980: 390, Fig. 1.10; 1981: 96, Fig. 1; 104: Fig.3) no se asocian con cuerpo central alguno, elemento básico del diseño en U. Este hecho fue percibido por Williams (1981: 103) al referirse a este lugar y señalar "aunque la estructura piramidal del fondo de la U no se ha incorporado claramente a la composición”. En tal sentido, el esquema evolutivo de Williams no se ajusta claramente a Paraíso.

b.- Al oeste y alejado de los montículos paralelos II y VI existen varios grupos destacando la Unidad I así como depósitos de basura que corresponderían a un asentamiento doméstico (Engel 1967: 247). Esta Unidad fue extensivamente excavada y restaurada por Engel (1966), distinguiéndose por numerosos compartimientos aglutinados y conectados por corredores. Se hallaron 5 y hasta 6 fases de construcción, para lo cual a cada reconstrucción precedía una etapa de rellenado de los recintos permitiendo levantar el nivel o altura del complejo. Los fechados radiocarbónicos arrojaron para la reconstrucción de menor antigüedad 3570 \pm 150 años, ubicándola en el Precerámico Tardío, con una antigüedad promedio de
2000 años a.C. Lo particular de la Unidad I es que al compararla con Huaca de los Reyes (Watanabe 1976), Garagay (Ravines 1975), se observan rasgos que la separan como una composición diferente. Además y en concordancia con lo indicado, existe en esta Unidad un recinto cuadrangular y un área central semihundida (Engel 1967), que tendría lejanos parecidos con el Templo de las Manos Cruzadas de Kotosh (Izumi y Sono 1963, Izumi 1971) y que responde a una tradición diferente (Burger y Salazar 1980).

c. - Otra de nuestras observaciones se refiere a la naturaleza funcional de los cuerpos laterales II y VI y el sitio en su conjunto. Williams (1980: 410; 1981: 101) sostiene que estos fueron habitacionales. $\mathrm{Al}$ respecto, Engel (1966) sostenía que los edificios se emplearon como viviendas aún cuando reconoce no saber el uso que dieron los habitantes a los restantes grupos de construcciones. Indica que su ubicación se relacionaría con las posibilidades agrícolas que ofrece el valle. Para Moseley (1975: 99) este sitio es compatible con "la circulación de las personas entre numerosos cuartos y compartimientos de forma generalmente similar; pudiendo producirse actividades diferentes y más o menos independientes en contextos espacialmente separados".

Ahora bien, aunque se asumiera que II y VI fueron habitacionales no puede asegurarse que ambos se construyeron y crecieron simultáneamente. Por otro lado, a pesar que las apreciaciones de Moseley son coherentes, ellas deben ser plenamente verificadas. Consecuentemente, en el presente estado de conocimientos la información no es suficiente para acercarnos al entendimiento del carácter funcional y socio-económico del lugar, a no ser que orientemos nuestros esfuerzos a examinarlo integralmente.

Pero Chuquitanta acoge otras interrogantes. Si bien parte del sitio ha sido asignado al Precerámico Tardío -conforme a los resultados de la Unidad I- lapso en que la sociedad se caracteriza por un sistema de vida sedentaria, con conocimientos del cultivo y manejo de las plantas, la explotación de los recursos marinos y la 
cría de animales, poco es lo que se sabe sobre el resto del yacimiento. De lograrse esta meta se fijaría sobre pilares más firmes la magnitud no solo formal del sitio sino también el proceso de su crecimiento. Pero lo cronológico no es todo. Este gran complejo requiere de una estrategia que analice su naturaleza interna y externa.

\section{BIBLIOGRAFIA}

BURGER, Richard and Lucy SALAZAR

1980 "Ritual and Religion at Huaricoto". En Archaeology, November - December, págs. 26-32. New York.

CHILDE, V. Gordon

1972 Introducción a la Arqueología. Ediciones Ariel, S.A. Barcelona.

ENGEL, Frédéric

1966 "Le Complexe Précéramique d’El Paraiso (Pérou)". En Journal de la Société des Américanistes. París, Tome LV-I, págs. 43-95.

1967 "El Complejo El Paraíso en el Valle del Chillón, habitado hace más de 3500 años; nuevos aspectos de la civilización de los agricultores del pallar". En Anales Científicos de la Universidad Agraria, Vol. V, julio-diciembre, $\mathrm{N}^{\circ} 3-4$, pp. 241-280. La Molina.

FLANNERY, Kent V.

1976 "The Early Mesoamerican House". En The Early Mesoamerican Village, (Kent V. Flannery Editor) pp. 16-24. Academic Press, Inc. New York.

FRITZ, John M.

1978 "Paleopsychology Today: Ideational Systems and Human Adaptation in Prehistory". En Social Archaeology. Beyond Subsistence and Dating. pp. 37-57. Academic Press Inc. New York.

IZUMI, Seiichi and Toshihiko SONO

1963 Andes 2. Excavations at Kotosh, Peru, 1960. Tokyo.

IZUMI, Seiichi

1971 "Development of Hre Formative Cultive in Hre Ceja de Montaña of. The Central Andes". En Dumbarton Oaks Conference on Chavin, October 26 th and 27 th. 1968. Dumbarton Oaks Research Library and Collection, Washington D.C.

LANNING, Edward P.

1967 Peru Before the Incas. Prentice-Hall, Inc., Englewood Cliffs, New Jersey.

LATHRAP, Donald, Jorge MARCOS and James

\section{ZEIDLER}

1977 "Real Alto: An Ancient Ceremonial Center". En Archaeology, Volume 30, Number 1, pp. 2-13. New York.

LUMBRERAS, Luis G.

1970 Los templos de Chavín. Guía para el visitante. Corporación Peruana del Santa. Lima.

MOSELEY, Michael E.

1975 The Maritime Foundations of Andean Civilizations. Cummings Publishing Company, Menlo Park, California.

RAVINES, Rogger

1975 "Garagay: un Viejo templo en los Andes". En Textual 10. pp. 6-12. Revista del INC. Lima.

RAVINES, Rogger y William H. ISBELL

1976 "Garagay: sitio ceremonial Temprano del valle de Lima". En Revista del Museo Nacional, Tomo XLI, 1975, pp. 253-275. Lima.

ROWE, John H.

1967 "Urban Settlements in Ancients Peru". En Peruvian Archaeology, Selected Readings (Rowe and Menzel Editor), pp. 293. 320. Peek Publications, Palo Alto, CA.

SCHAEDEL, Richard P.

1951 "Major Ceremonial and Population Centers in Northern Peru". En Civilizations of Ancient America; Selected Papers of the XXIX. International Congress of Americanists. pp. 232-243. University of Chicago Press. Chicago.

SCHEELE, Harry G.

1970 The Chavin Occupation of the Central Coast of Peru. Tesis de Doctor. Universidad de Harvard, EE.UU.

TRIGGER, Bruce G.

1968 "The Determinants of Settlements Patterns". En Settlement Archaeology (K.C. Chang Editor) pp. 53-78. National Press Books, Palo Alto, California.

WATANABE, Luis

1976 Sitios Tempranos en el valle de Moche (Costa Norte del Perú). Tesis doctoral. Programa Académico de Ciencia Social, especialidad Arqueología, Universidad Nacional Mayor de San Marcos, Lima.

WILLEY, Gordon R.

1953 "Prehistoric Settlement Pattern in the Virú Valley, Perú". Smithsonian Institution Bureau of American Ethnology Bulletin 155. Washington D.C. 
1971 An Introduction to American Archaeology. Volume Two: South America. PrenticeHall Inc., Englewood Cliffs, New Jersey.

WILLIAMS LEON, Carlos

1971 "Centros ceremoniales tempranos en los valles del Chillón, Rímac y Lurín”. En Apuntes Arqueológicos 1, pp. 1-4. Lima.

1972 "La difusión de los pozos ceremoniales en la costa peruana”. En Apuntes Arqueológi$\cos 2$. Lima.

1980 "Arquitectura y Urbanismo en el Antiguo Perú”. En Historia del Perú, Tomo VIII, pp.
369-585. Juan Méjía Baca, Editor. Lima.

1981 "Complejos de pirámides con planta en U, patrón arquitectónico de la costa central". En Revista del Museo Nacional, Tomo XLIV, 1978-1980, pp. 95-100. Lima.

WINTER, Marcus C.

1976 "The Archaeological Household Cluster in the Valley of Oaxaca". En The Early Mesoamerican Village. K.V. Flannery Editor. pp. 25-30. Academic Press, Inc., New York.

$77^{\circ}$

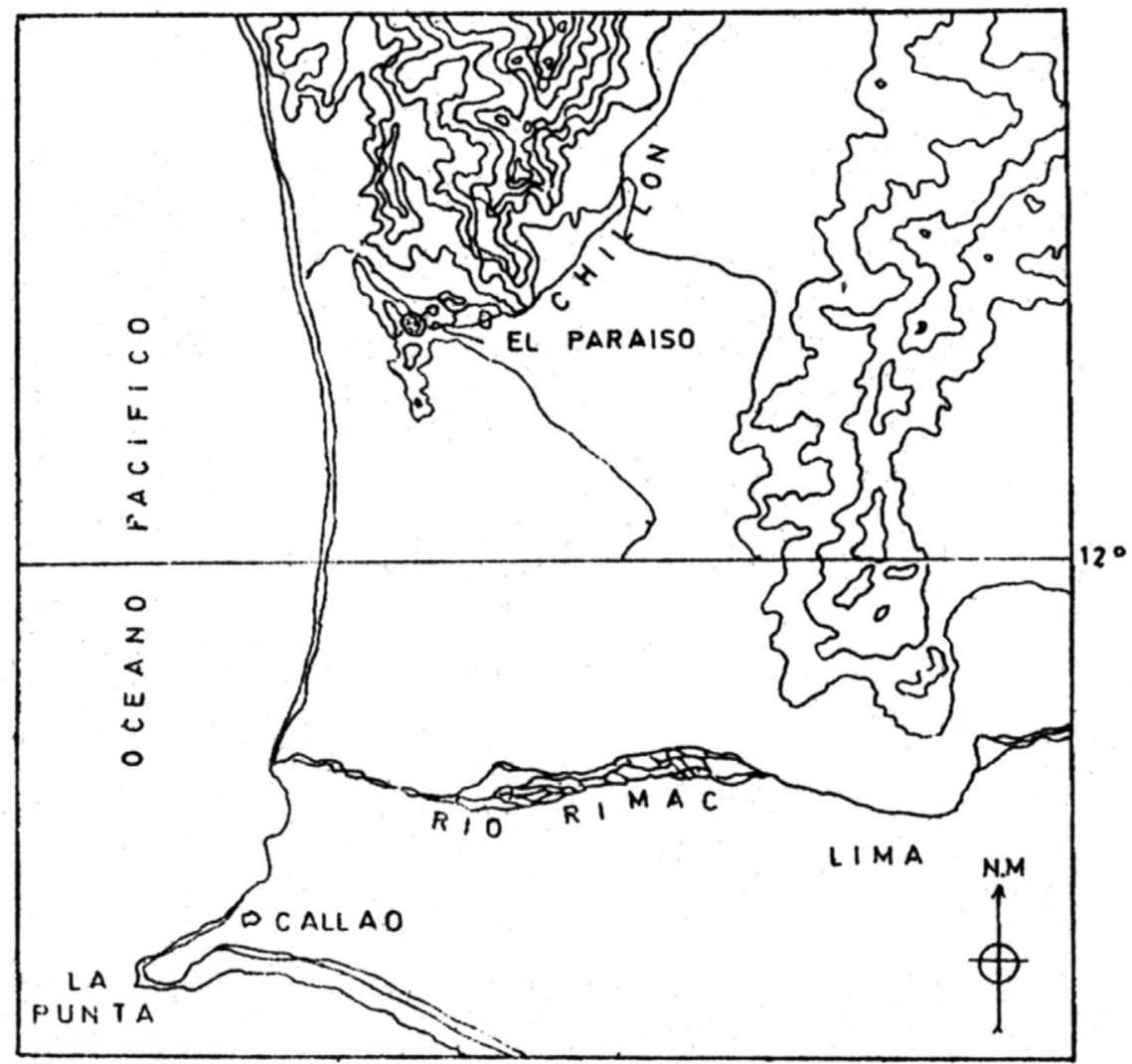

770

Fig. 1: Ubicación deEl Paraíso (tomado de Engel 1,966) 